\title{
The Potential Protective Impact of Spirulina on Cisplatin Induced-Renal Toxicity in Adult Male Albino Rats: Histological, Immunohistochemical and Biochemical Study \\ Hala Taha Shalan and Yasmin Ramadan Abd El Fattah
}

Anatomy and Embryology department, Ain Shams University, Faculty of medicine, Cairo, Egypt

Corresponding author: Hala Taha Shalan, Email: halashalan1986@yahoo.com, Orcid id:0000-0002-0067-4764, Mobile:00201001970159

\begin{abstract}
Introduction: Cisplatin (CP) is regarded as a prevalent anticancer medication utilized against several cancer types. Spirulina (SP), a blue-green alga, has been utilized as a nutritional supplementation, in addition to other therapeutic enforcement. Spirulina includes lipids, proteins, carbohydrates, and vitamins including a pigmented protein and b-carotene besides some vital minerals.

Objectives: The present study was carried out to verify the toxic impacts of cisplatin on kidney of the rat and the probable protective role of spirulina platensis supplementation.

Material and methods: The twenty-four rats were randomly categorized into three equal groups (eight rats/each). Group I (Control Group): Eight rats were subdivided into two subgroups, four rats each: Group I-a: Four rats did not undergo any experiments and received only food and water for 8 days. Group I-b: Four rats received Spirulina $(500 \mathrm{mg} / \mathrm{kg})$ body weight orally by using a gastric tube for 8 days. Group II: The animals were administrated a single dose of cisplatin $6 \mathrm{mg} / \mathrm{kg}$ body weight intra-peritoneal. Group III: Each rat received Spirulina $(500 \mathrm{mg} / \mathrm{kg})$ body weight orally for 8 days and cisplatin 6 $\mathrm{mg} / \mathrm{kg}$ body weight intraperitoneal on day 4 .

Results: cisplatin showed extensive tubular damage as wide vacuolations in tubular epithelium, pyknotic nuclei and distortion of renal corpuscles showing widening of Bowman's space with adherence of glomerular capillary tuft to the parietal layer of Bowman's capsule. Administration of spirulina showed regeneration of tubular epithelium and presence of tubular brush border and apparently normal lumen.

Conclusion: Cisplatin resulted in histological as well as biochemical changes in the kidney of adult male rats. Administration of spirulina with cisplatin attenuated these negative impacts which can be attributed to the antioxidant activity.
\end{abstract}

Keywords: Cisplatin, Spirulina, Renal tissue, Caspase 3.

\section{INTRODUCTION}

Cisplatin (CP) is regarded as a prevalent anticancer medication utilized against several cancer types as cervical, uterine, ovarian, and testicular malignancies. Furthermore, it is believed to be toxic to the kidney, ear, gastrointestinal tract, and nervous system. This drug is believed to be biologically active to a nephrotoxic substance ${ }^{(\mathbf{1}, \mathbf{2})}$. After cisplatin treatment about $20-30 \%$ of cases, experience acute kidney injury (AKI) in spite of the therapeutic improvements. Cases that experience AKI have an elevated mortality risk and probably encounter chronic kidney injury (CKI) ${ }^{(3,4)}$.

Nephrotoxicity mainly occurs in the epithelial cells of proximal renal tubule. The concentration of cisplatin in the cells of epithelial tubular is five times more elevated compared to its concentration in the blood. Renal dysfunction happens after days from administrating cisplatin $\left(50-120 \mathrm{mg} / \mathrm{m}^{2}\right)$ in therapy. Proximal as well as distal convoluted tubules are the main target for cisplatin impacts in the kidney, where it induces oxidative stress, cellular damage, DNA damage, nephritis, and apoptosis $(5,6)$.

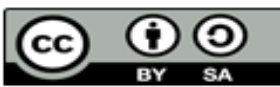

This article is an open access article distributed under the terms and conditions of the Creative Commons Attribution (CC BY-SA) license (http://creativecommons.org/licenses/bv/4.0/)
Nephrotoxicity triggered by cisplatin is because of reactive oxygen species (ROS) formation. Cisplatin also leads to the inhabitation of several antioxidant enzymes like glutathione-S-transferase (GST), glutathione peroxidase (GPX), as well as superoxide dismutase (SOD). This leads to mitochondrial lysis and generation of ROS in cells ${ }^{(7)}$. Generation of ROS induces cascades of signal transduction, which leads to necrosis and apoptosis. To date, the mechanism of cisplatin-induced ROS signaling pathways remains undetermined, as there is no treatment until now to retrain cisplatin side effects. Multiple agents have demonstrated a nephroprotective impact in renal damage models caused by cisplatin and it is clearly evident that most of them are antioxidants ${ }^{(\mathbf{8})}$.

Spirulina (SP), a blue-green alga, was utilized as a nutritional supplementation in addition to some therapeutic enforcement. Spirulina includes lipids, carbohydrates, proteins, vital minerals, vitamins like a pigmented protein and $b$-carotene. The antioxidant effect of spirulina has been proven in vitro as well as in vivo studies. Spirulina is recognized due to its broad biological 
activity, and antimutagenic, anti-inflammatory, immune boosting and cardioprotective features ${ }^{(\mathbf{9 - 1 1})}$.

Spirulina platensis is a unicellular cyanobacterium which has been broadly utilized as a dietary supplementation because of its nutritional value. It contains powerful antioxidants and free radical scavenging agents ${ }^{(\mathbf{1 2})}$. It has been significantly used in preclinical studies, as in anemia prevention, antiviral and antimicrobial reduction of HIV replication velocity, immunostimulant, and anti-carcinogenic antioxidant, in addition to contributing to the prevention of poisoning by heavy metals, antibody production stimulation as well as hepatoprotective, antigenotoxic and neuroprotective activities ${ }^{(13-15)}$. Spirulina is produced simply because of its reasonable growth requirements, its excellent conservation after recollection, as well as its security regarding consumption ${ }^{(\mathbf{1 6})}$.

The present study was carried out to verify the toxic impacts of cisplatin on kidney of the rat and the probable protective role of spirulina platensis supplementation.

\section{MATERIALS AND METHODS}

\section{Chemicals:}

Cisplatin was obtained from EIMC United

Pharmaceuticals, Egypt. The drug was administered intraperitoneal.

Spirulina tablets were purchased from Puritan's Pride Company, Inc. (USA). Each tablet contains $500 \mathrm{mg}$ of the active ingredient. The drug was ground and administrated orally as a new suspension in $0.9 \%$ saline.

Animals: The current study was performed on twentyfour adult albino rats of local strain, their age ranged between 3-5 months and their weight ranged between (180-220g). The rats were purchased from the Medical Ain Shams Research Institute (MASRI). Rats were housed for one week for environmental adaptation according to standard laboratory conditions on a twelvehour light/dark cycle. They received an adequate and constant diet along with free access to water ad libitum. Rats were kept isolated for two weeks in a laboratory room at comfortable room temperature for adaptation before any experiments. Moreover, all experiments have been conducted at the same time of the day, between 8 a.m. and 2 p.m., in order to overcome variations because of diurnal rhythms.

\section{Ethical Consideration:}

All the experiments were performed based on the guidelines issued by the Animal Research Ethics Committee of Faculty of Medicine, Ain Shams University. The procedure was performed based on the Ethical Guidelines for diagnosing experimental pain in conscious animals ${ }^{(17)}$.
Experimental Protocol: The 24 rats were randomly categorized into three equal groups ( 8 rats/each):

Group I (Control Group): Eight rats which were subdivided into two subgroups, four rats each:

Group I-a (negative control): Four rats did not undergo any experiments, and received only food and water for 8 days.

Group I-b (positive control): Four rats received spirulina $(500 \mathrm{mg} / \mathrm{kg})$ body weight orally by using a gastric tube for 8 days ${ }^{(\mathbf{1 8})}$.

Group II (cisplatin-treated group): The animals were administrated a single dose of cisplatin $6 \mathrm{mg} / \mathrm{kg}$ body weight intraperitoneal ${ }^{(\mathbf{1 9})}$.

Group III (cisplatin + spirulina-treated group): Each rat received spirulina $(500 \mathrm{mg} / \mathrm{kg})$ body weight orally for 8 days and cisplatin $6 \mathrm{mg} / \mathrm{kg}$ body weight intraperitoneal on day 4.

Before scarification of rats, blood was collected from the tail veins of animals belonging to each group for assessment of plasma urea, albumin and creatinine levels. Midline incision was made in the abdomen, while kidneys were isolated. All rats were sacrificed through cervical dislocation after light ether anesthesia ${ }^{\mathbf{2 0})}$.

\section{Biochemical assays}

Plasma urea, albumin and levels of creatinine were computed spectrophotometrically by commercially available kits.

\section{Histological studies}

With respect to the light microscopy study, specimens were put in $10 \%$ neutral buffered formalin, dried, fixed in paraffin, and subsequently sections were cut at $5 \mu \mathrm{m}$ and stained with hematoxylin and eosin (H\&E) for a routine histological examination with Masson's trichrome stain to assess the amount of collagen deposition ${ }^{(21)}$.

Immunohistochemical stain for caspase-3 (indicator of apoptosis): Sections were rinsed in phosphate buffered saline for $5 \mathrm{~min}$, and subsequently incubated with caspase-forming antibody with a 1:200 dilution (Invitrogen, Sweden AB Stockholm Sweden) overnight at $4^{\circ} \mathrm{C}$. Sections were then rinsed and incubated with a secondary goat and rabbit antibody ((1:500) Invitrogen, Molecular Probes, Eugene, Oregon, USA) for $1 \mathrm{~h}$ at room temperature. Slides were incubated in 3, 3diaminobenzidene for $10 \mathrm{~min}$ and then counterstained by Mayer's hematoxylin, dehydrated and mounted by dibutyl phthalate in xylene ${ }^{(22)}$. Paraffin-stained sections were photographed after examination by light microscope (Olympus 268M microscope) equipped with an automatic photomicrographic camera system. 


\section{Statistical analysis}

Analysis of data was carried out via the Statistical Package for Social Science (IBM Corp, released 2013. IBM SPSS statistics for windows, V. 22.0. Armonk, NY. USA). Parametric quantitative data were expressed as mean \pm standard deviation (SD). One-way analysis of variance (ANOVA) test was utilized to compare the variance of parametric continuous variable among the independent categorical variables with three levels. Post Hok Tukey test was used for comparison between the levels of the categorical independent variables. All $\mathrm{P}$ values were two-tailed, the probability (P-value) of $>0.05$ was regarded statistically as non-significant, $\leq 0.05$ was statistically significant and $\leq 0.01$ was highly statistically significant. Data in the present study were expressed as Mean \pm standard error of the mean (SEM).

\section{RESULTS \\ Histological results: \\ Groups I (Control group)}

In the present work, no substantial differences have been detected between the subgroups of group I, therefore the results of two subgroups will be discussed together.

$\mathrm{H} \&$ E-stained section, light microscopic examination of the renal gland of group I (control group) demonstrated the histological structure of the gland. The renal cortex appeared formed of distal convoluted tubules, renal corpuscles, proximal convoluted tubules, as well as cortical collecting tubules. Each corpuscle was composed of glomerulus made up of many capillary loops containing red blood cells and surrounded by Bowman's space between visceral and parietal layer of Bowman's capsule (Fig.1).

Masson's Trichrome stain demonstrated a minimal amount of collagen fiber in the renal interstitial and in between glomerular capillaries (Fig. 2).

Immunohistochemically-stained section, examination of caspase-3 showed normal kidney architecture and no apoptotic cells (Fig. 3).

\section{Groups II (cisplatin-treated group)}

Light microscopic examination of $\mathrm{H} \&$ E-stained sections of the renal gland of group II (cisplatin-treated group) showed extensive tubular damage as wide vacuolations in tubular epithelium, pyknotic nuclei and distortion of renal corpuscles showing widening of Bowman's space with adherence of glomerular capillary tuft to parietal layer of Bowman's capsule. Note illdefined, irregular outer boundary of the renal corpuscle and apparent hypercellularity and vascular congestion of capillary tuft (Fig. 4, 5).

Masson's Trichrome stain showed an extensive amount of collagen fibers distributed mainly around the renal tubules and within the glomeruli (Fig. 6).

Examination of immunohistochemically-stained sections for caspase-3 showed that renal tubules were considered caspase- 3 activation main site in animals treated with cisplatin (Fig. 7).

\section{Groups III (cisplatin + spirulina-treated group)}

Light microscopic examination of $\mathrm{H} \&$ E-stained sections of the renal gland of group III showed regeneration of tubular epithelium and presence of tubular brush border and apparently normal lumen. Renal glomeruli showed normal cellularity and intact bowman's space (Fig. 8).

Masson's Trichrome stain showed minimal amount of the collagen (Fig. 9).

Examination of immunohistochemically-stained sections for caspase- 3 showed that spirulina substantially diminished the active caspase- 3 expression caused by cisplatin. There were only a few focal acute tubular necrosis areas as well as a few apoptotic cells that demonstrated brown cytoplasmic stains (Fig. 10).

\section{Morphometric and statistical results}

Histograms (1,2 and 3) demonstrate that cisplatin injection (intraperitoneal) at a $6 \mathrm{mg} / \mathrm{kg}$ single dose induced substantial elevation in creatinine, serum urea and a significant decrease in serum albumin level in comparison with the control group. In contrast, spirulina administration for 8 days with single dose of cisplatin on day 4 substantially diminished the elevated levels of creatinine and urea, in addition to a significant increase in the level of serum albumin in comparison with the group treated with cisplatin.

Table (1): ANOVA test for urea (mg/dl) comparing group II and III to the control group.

\begin{tabular}{|l|l|l|l|l|l|l|l|}
\hline & N & Mean & SD & Minimum & Maximum & F & Pvalue \\
\hline Control & 8 & 32.19 & 2.219 & 29 & 36 & 3783.530 & $<0.001^{*}$ \\
\hline Cisplatin & 8 & 115.75 & 2.605 & 112 & 120 & & \\
\hline Cisplatin + Spirulina & 8 & 37.25 & 1.488 & 35 & 39 & & \\
\hline
\end{tabular}

*Significant 
Table (2): Tukey Post Hoc test for urea (mg/dl) comparing control group to group II and III, group II to group III.

\begin{tabular}{|l|l|l|}
\hline Group & Group & Sig. \\
\hline Control & Cisplatin & $<0.001^{*}$ \\
\hline Control & Cisplatin + Spirulina & $<0.001^{*}$ \\
\hline Cisplatin & Cisplatin + Spirulina & $<0.001^{*}$ \\
\hline
\end{tabular}

*Significant

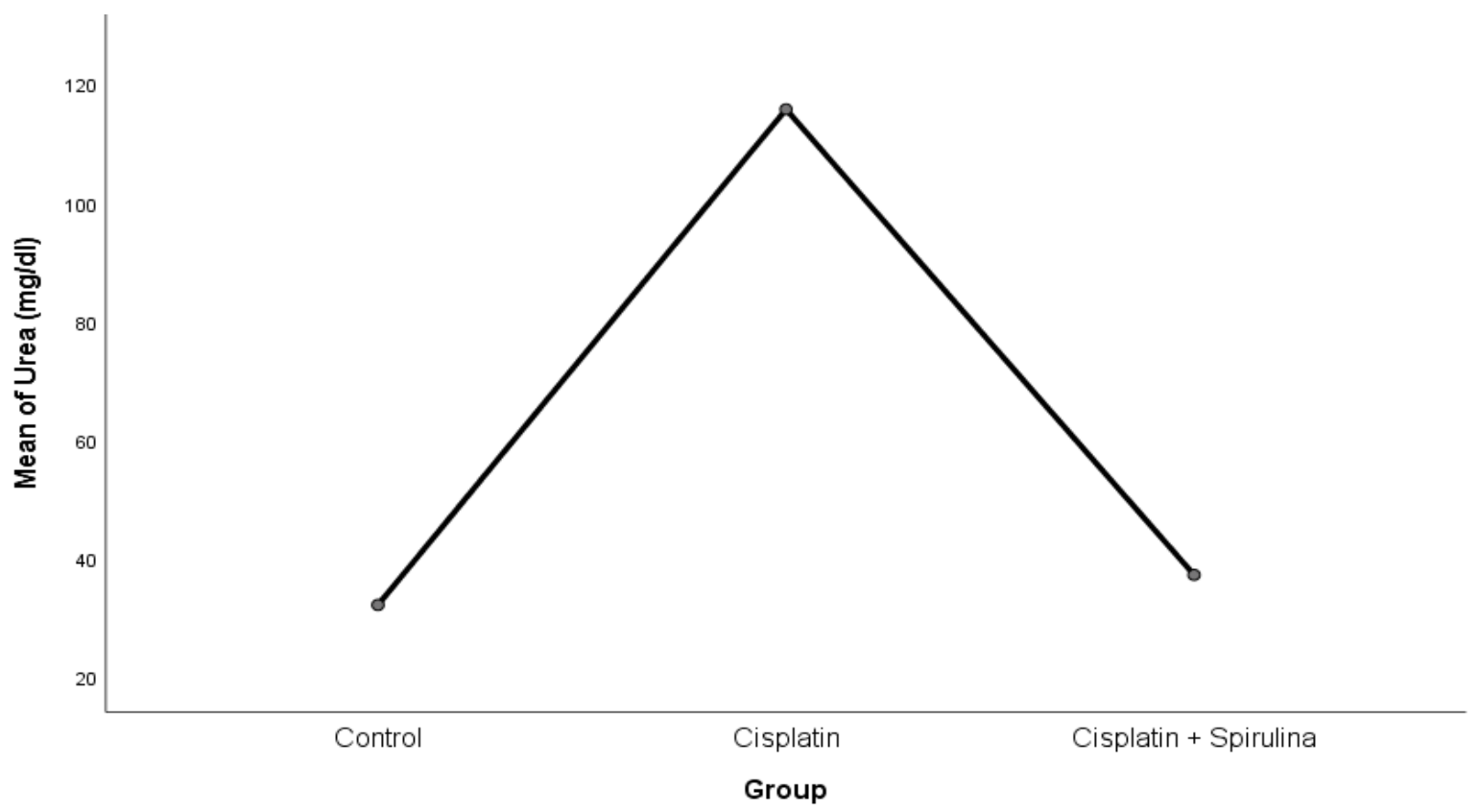

Histogram (1): Means Plots graph for urea among different groups.

Table (3): ANOVA test for creatinine (mg/dl) comparing group II and III to the control group.

\begin{tabular}{|c|c|c|c|c|c|c|c|}
\hline & N & Mean & SD & Minimum & Maximum & F & Pvalue \\
\hline Control & $\mathbf{8}$ & $\mathbf{. 7 5 2}$ & $\mathbf{. 0 1 8}$ &. $\mathbf{7 2}$ & .77 & $\mathbf{3 8 4 6 . 2 7 1}$ & $<\mathbf{0 . 0 0 1 *}$ \\
Cisplatin & $\mathbf{8}$ & $\mathbf{3 . 3 4 3}$ & $\mathbf{. 0 4 6}$ & $\mathbf{3 . 3 0}$ & $\mathbf{3 . 4 2}$ & & \\
Cisplatin + Spirulina & $\mathbf{8}$ & $\mathbf{1 . 0 0 7}$ & $\mathbf{. 1 0 1}$ & $\mathbf{. 8 9}$ & $\mathbf{1 . 2 0}$ & & \\
\hline
\end{tabular}

*Significant

Table (4): Tukey Post Hoc test for creatinine (mg/dl) comparing control group to group II and III, group II to group III.

\begin{tabular}{|l|c|c|}
\hline \multicolumn{1}{|c|}{ Group } & Group & Sig. \\
\hline Control & & \\
\hline Control & Cisplatin & $<\mathbf{0 . 0 0 1 *}$ \\
\hline Cisplatin & Cisplatin + Spirulina & $<\mathbf{0 . 0 0 1 *}$ \\
\hline
\end{tabular}

*Significant 


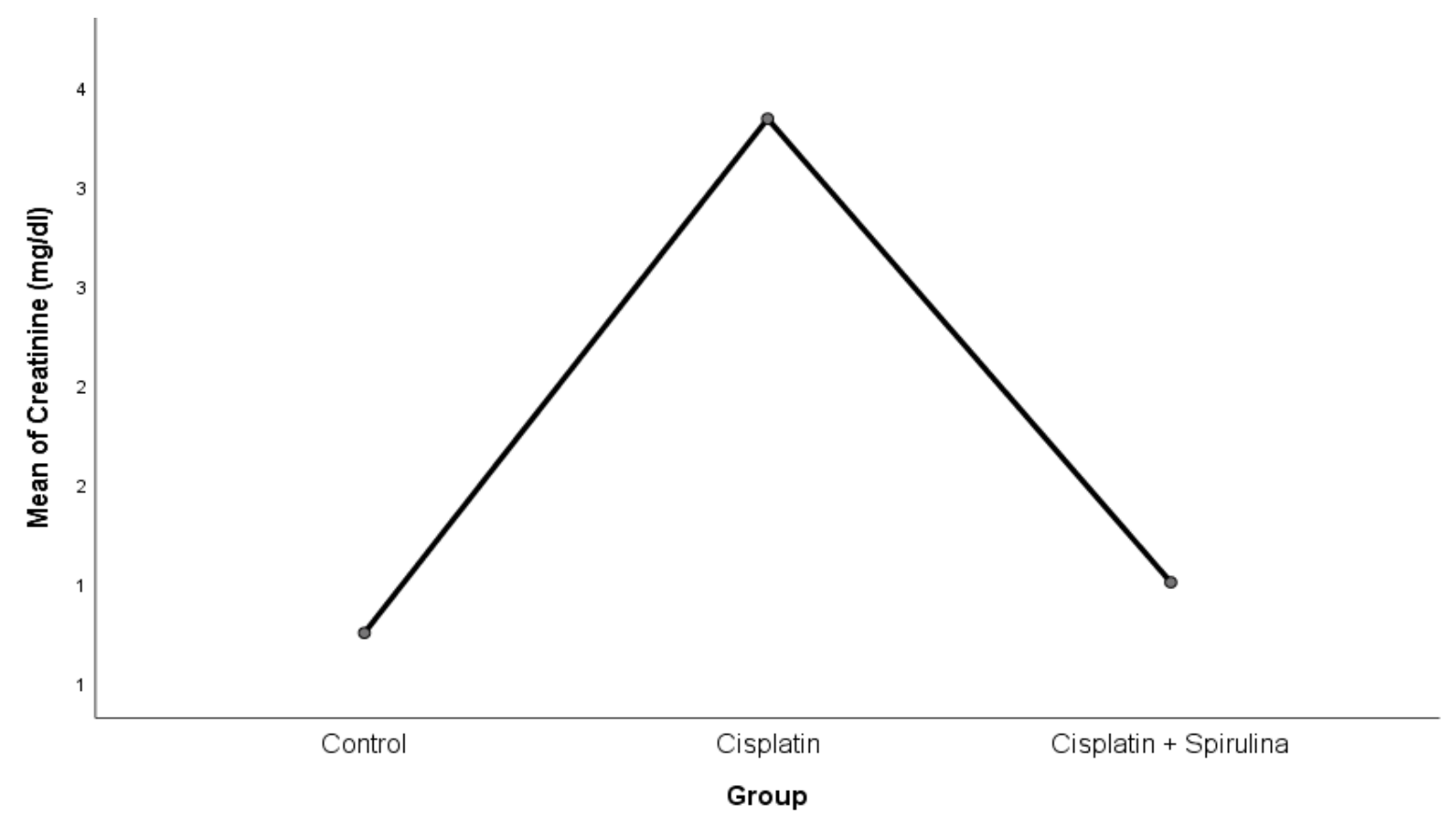

Histogram (2): Means Plots graph for creatinine among different groups.

Table (5): ANOVA test for albumin (g/dl) comparing group II and III to the control group.

\begin{tabular}{|l|l|l|l|l|l|l|l|}
\hline & $\mathrm{N}$ & Mean & SD & Minimum & Maximum & F & Pvalue \\
\hline Control & 8 & 4.233 & .166 & 3.90 & 4.50 & 129.441 & $<0.001 *$ \\
Cisplatin & 8 & 2.451 & .289 & 1.90 & 2.90 & & \\
Cisplatin + Spirulina & 8 & 3.775 & .219 & 3.50 & 4.10 & & \\
\hline
\end{tabular}

\section{*Significant}

Table (6): Tukey Post Hoc tests for albumin (g/dl) comparing control group to group II and III, group II to group III.

\begin{tabular}{|l|l|l|}
\hline Group & Group & Sig. \\
\hline Control & & \\
\hline Control & Cisplatin & $<\mathbf{0 0 0 1} *$ \\
\hline Cisplatin & Cisplatin + Spirulina & $\mathbf{0 . 0 0 2}$ \\
\hline
\end{tabular}

*Significant

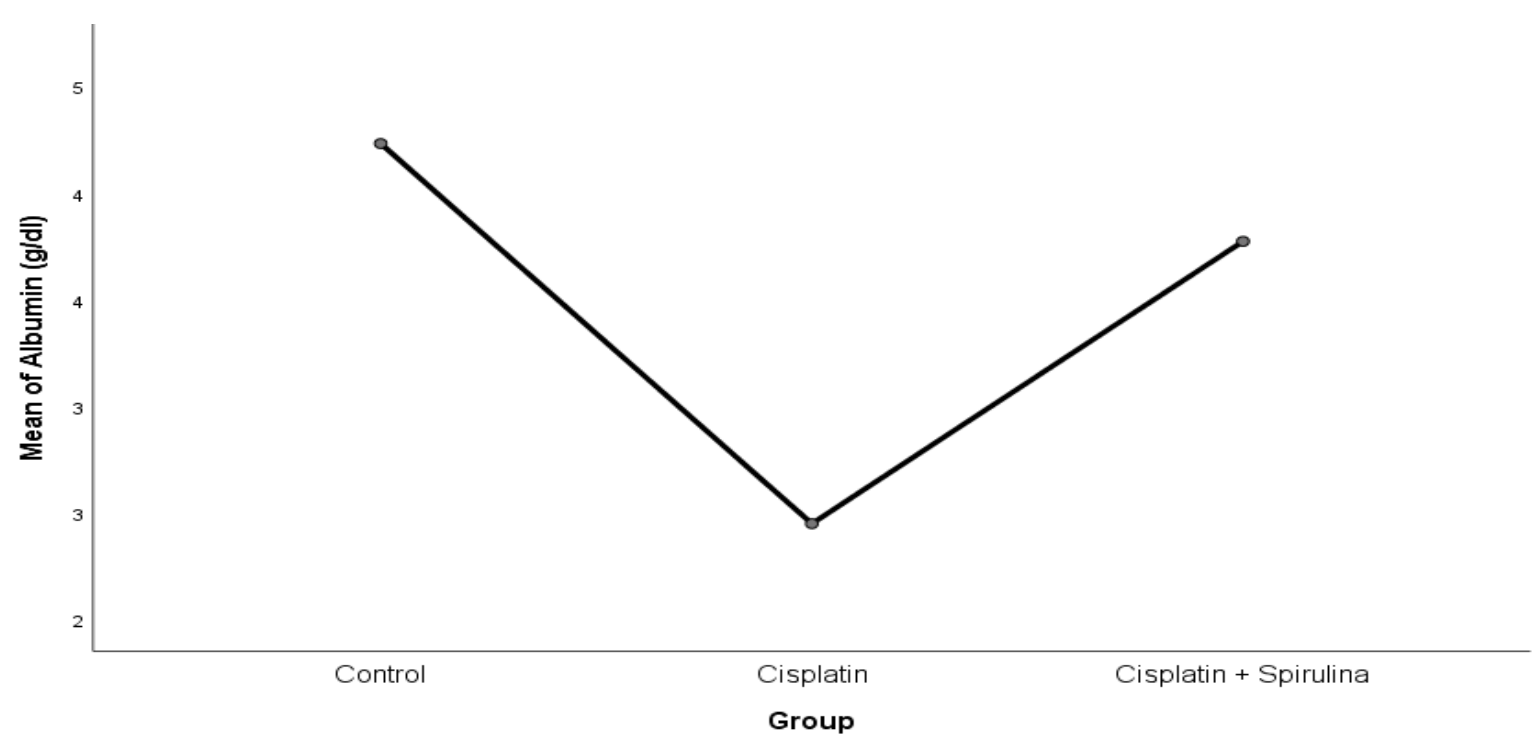

Histogram (3): Means Plots graph for Albumin among different groups 


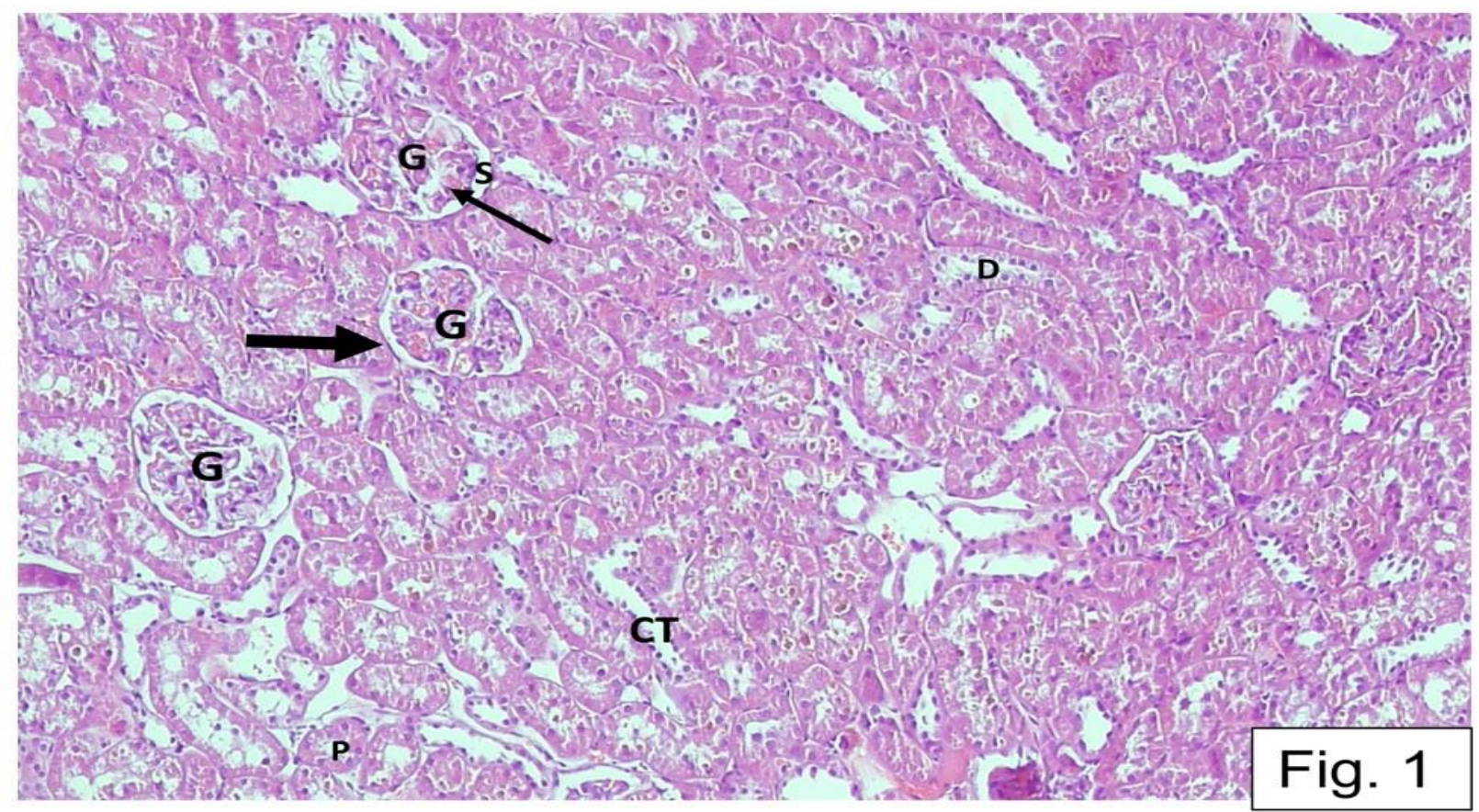

Fig. (1): A photomicrograph of a section of the renal cortex from group (I) control group detecting normal renal corpuscles (thick arrows) consisted of glomerular tuft of capillaries $(\mathrm{G})$ and subcapsular space (S). PCT (P) showed narrow lumen, and its lining cells had basal nuclei and deeply acidophilic cytoplasm. DCT (D) showed wide lumen, and its lining cells had apical nuclei and few acidophilic cytoplasm. Notice: the presence of collecting tubules (CT). (H \& E x 400)

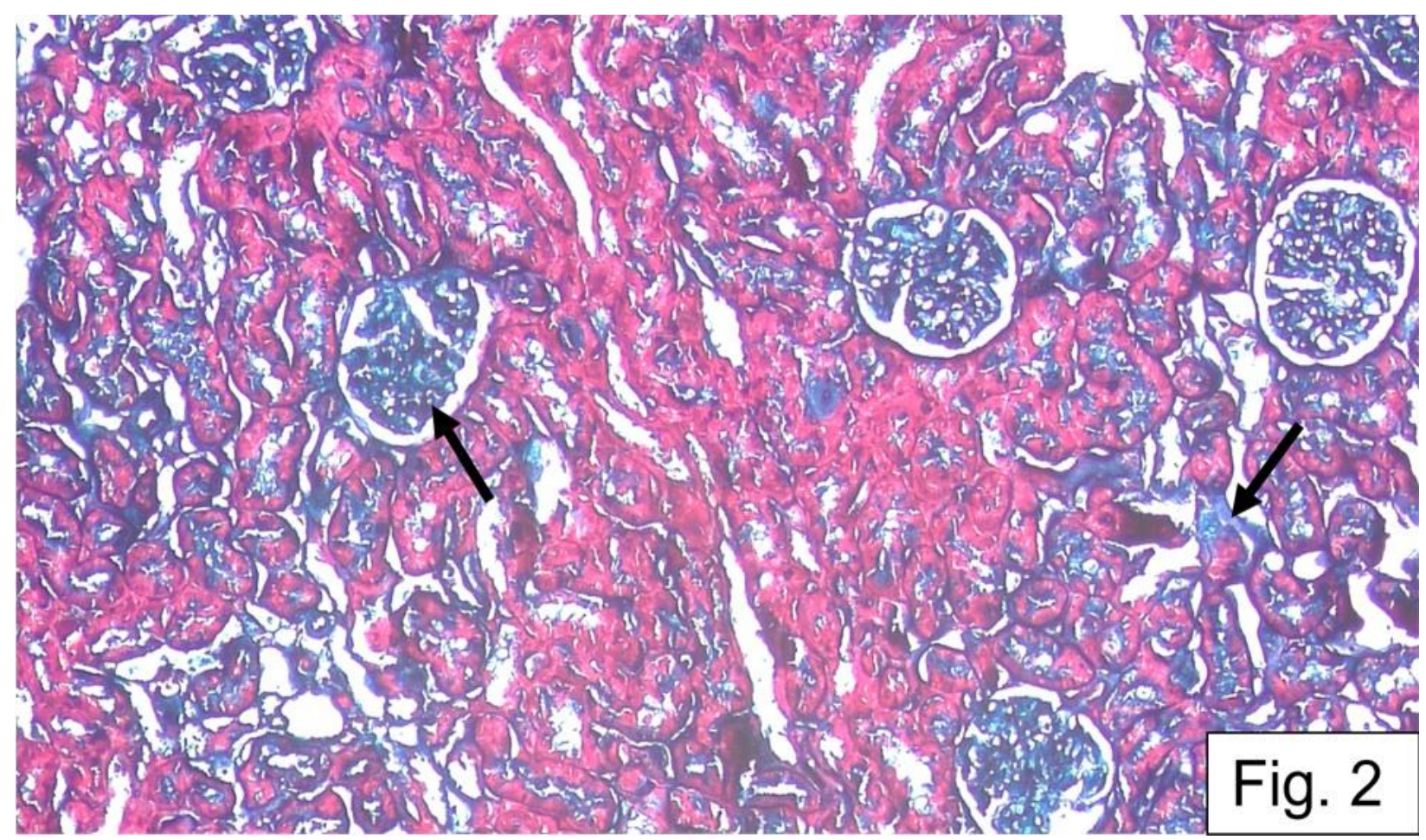

Fig. (2): A photomicrograph of a section of the renal cortex of group (I) control group representing minimal amount of the collagen fiber present in the renal interstitial and in between glomerular capillaries (arrows).

(Masson's Trichrome x 400) 


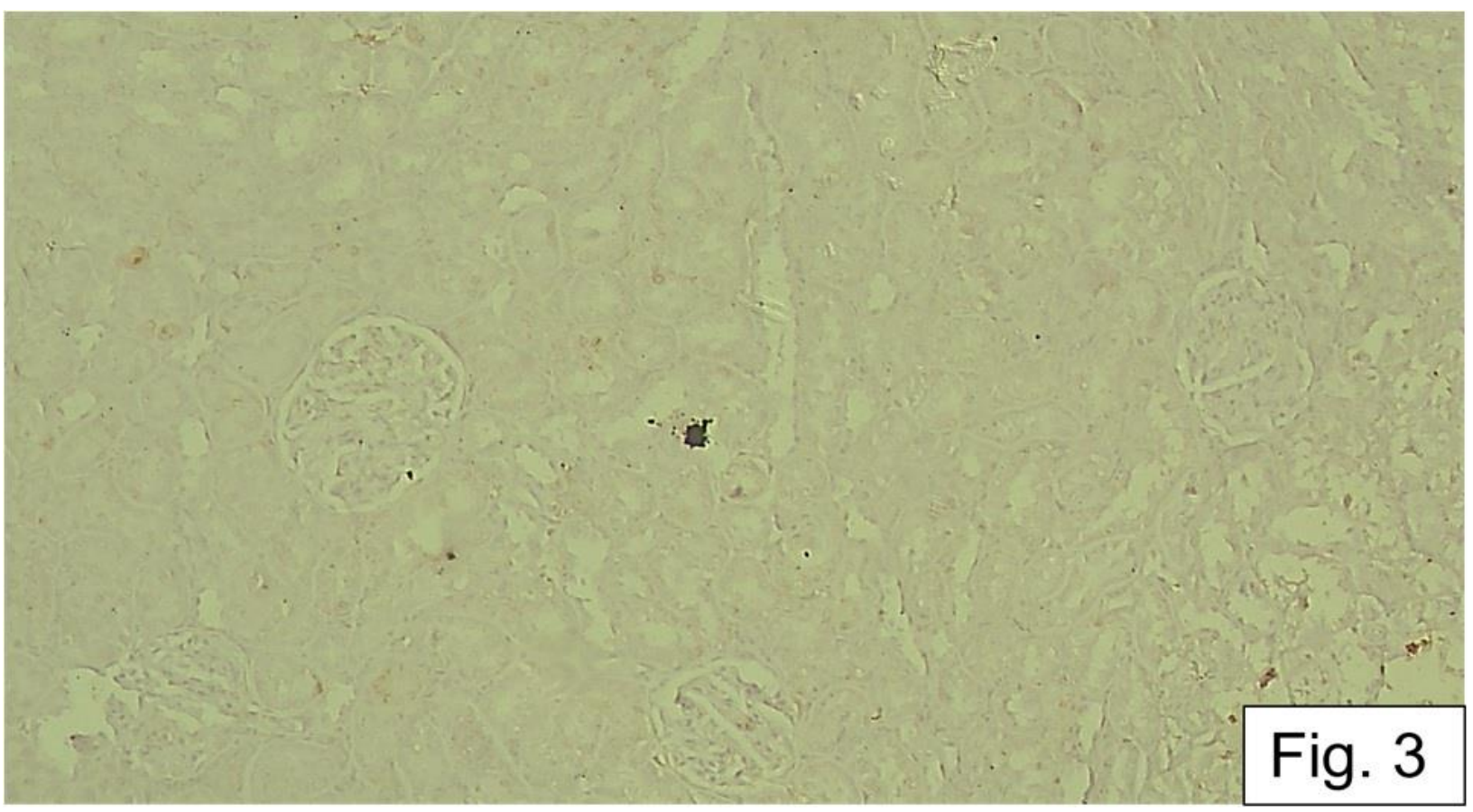

Fig. (3): A photomicrograph of a section of the renal cortex of group (I) control group detecting normal kidney architecture and no apoptotic cells. (Caspase-3 X400)

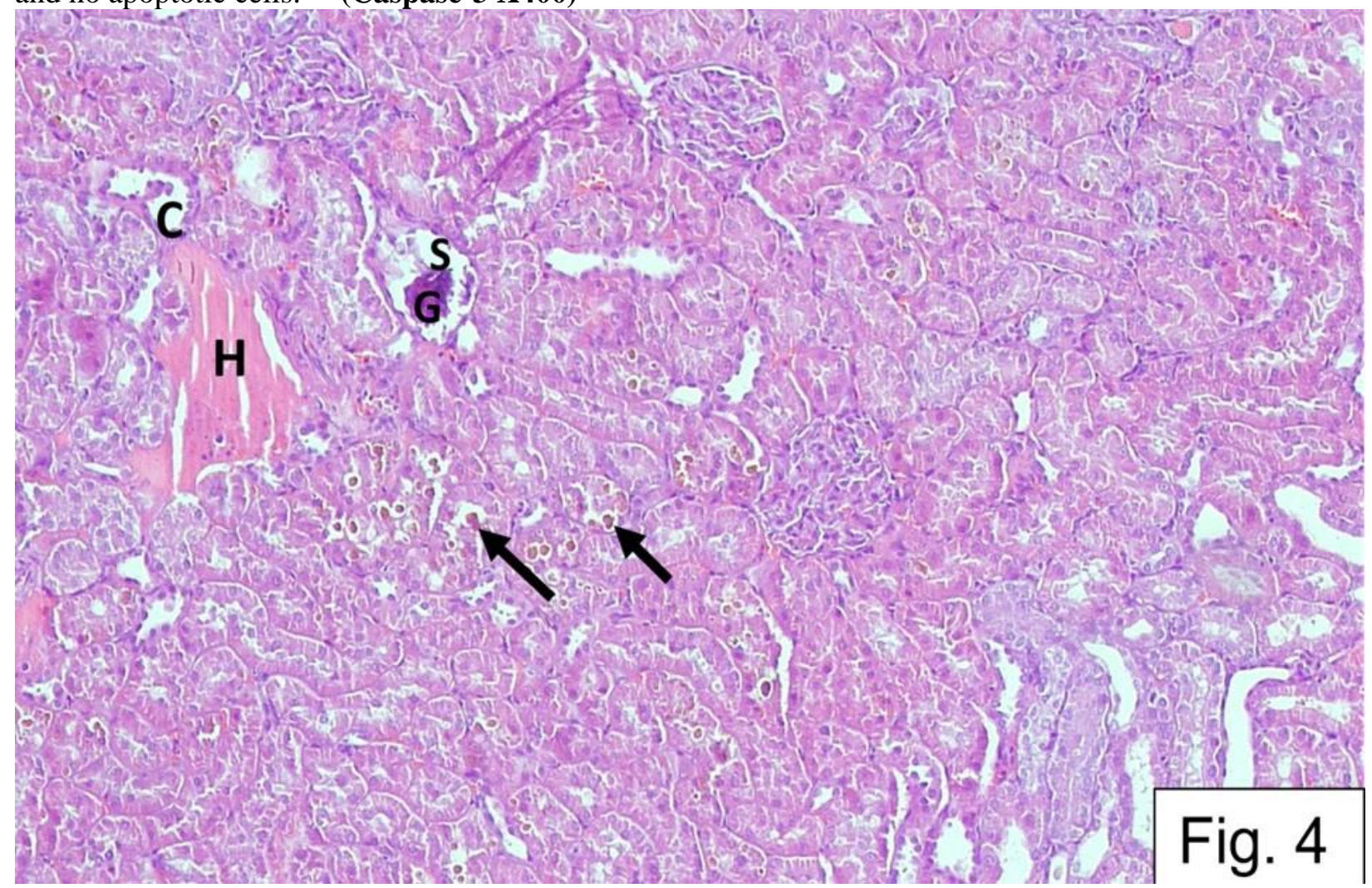

Fig. (4): A photomicrograph of a section of the renal cortex from group (II) cisplatin-treated group detecting renal corpuscle associated with atrophy in the glomerulus $(\mathrm{G})$ and widening of the subcapsular space (S). Some tubules show necrotizing epithelium (pyknosis) (thin arrow) and cystic dilatation (CS). Notice: presence of hemorrhage (H) in the interstitial. (H\&E x400) 


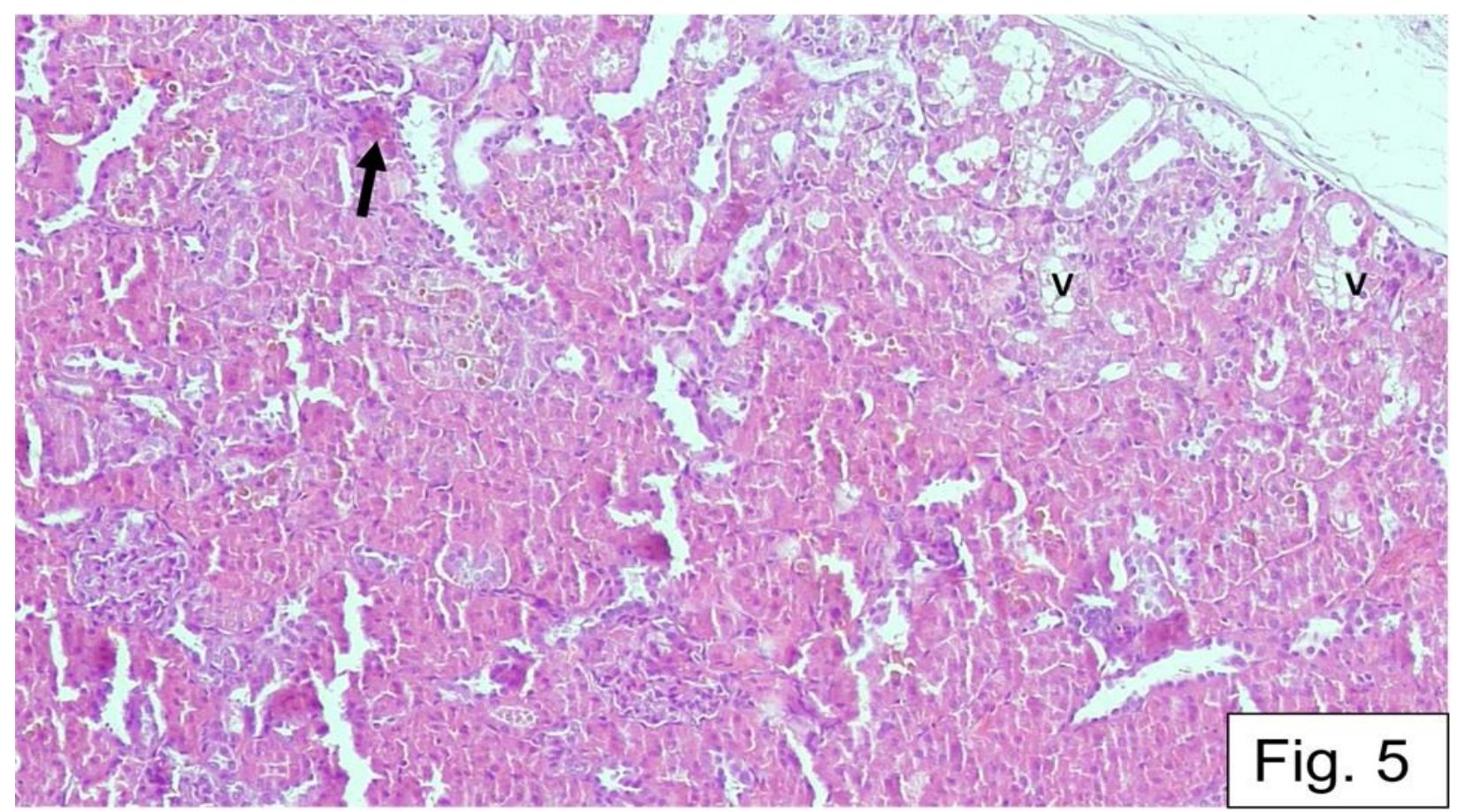

Fig. (5): A photomicrograph of a section of the renal cortex from group (II) cisplatin-treated group representing, hypercellularity with widening in the lumen. Proximal tubules showed vacuolation (V) of its epithelial cells and vascular congestion of capillary tuft (arrow). (H\&E x400)



Fig. (6): A photomicrograph of a section of the renal cortex of group (II) cisplatin-administered group showing extensive amount of the collagen fibers which is distributed mainly around the renal tubules and in between the glomeruli (arrows). (Masson's Trichrome $x$ 400) 


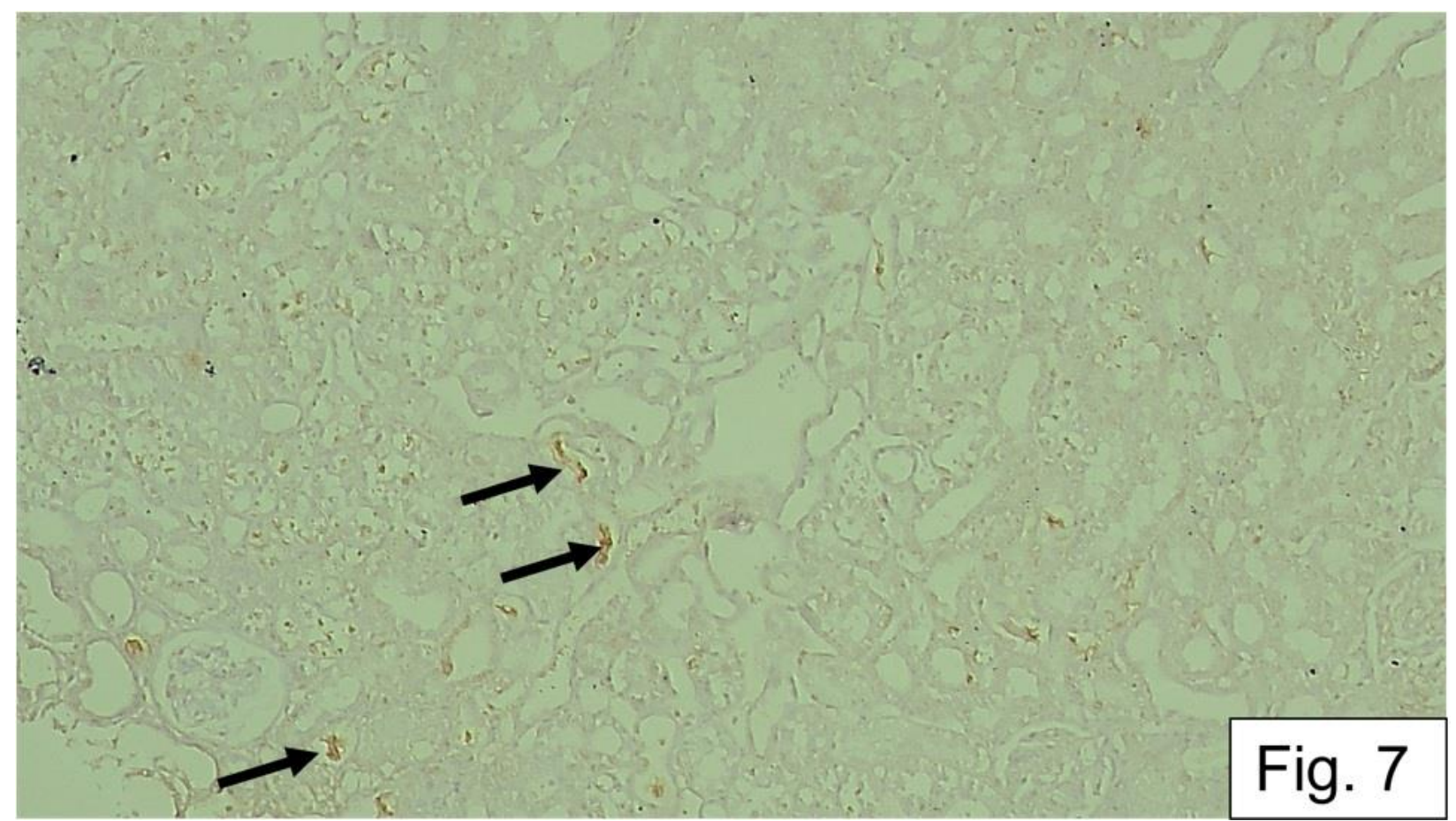

Fig. (7): A photomicrograph of a section of the renal cortex of group (II) cisplatin-treated group representing that, the renal tubules were the main site for caspase- 3 activation in cisplatin-administered animals with the presence of apoptotic cells appeared as brownish cytoplasmic staining (black arrow). (Caspase-3 X400)

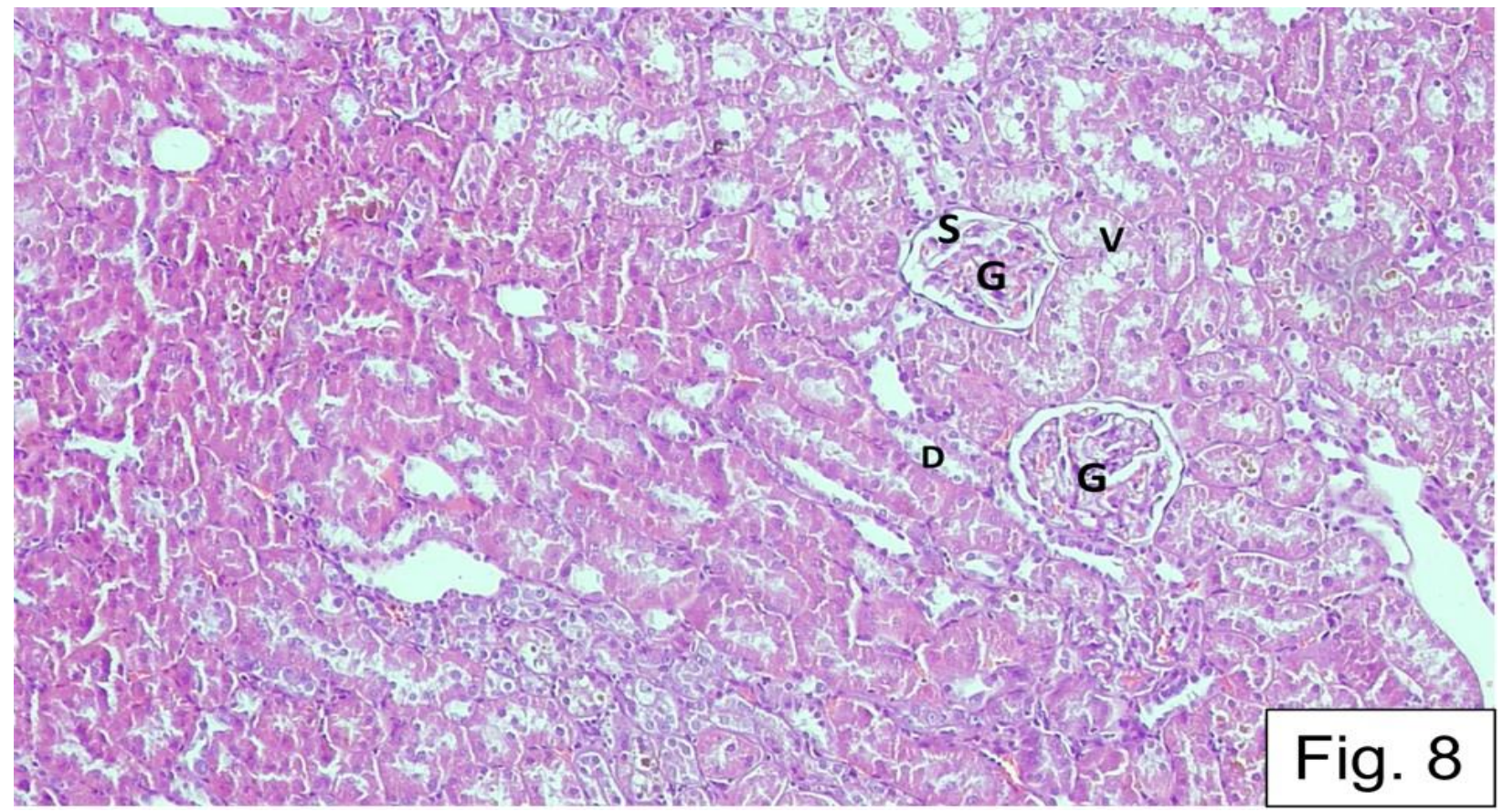

Fig. (8): A photomicrograph of a section of the renal cortex from group (III) (cisplatin+ spirulina-treated group) showing less tubular edema with restoration of normal architecture of the renal tubules, which was formed of glomerular tuft of capillaries $(\mathrm{G})$ with the presence of subcapsular space (S). Distal convoluted tubules (D) also detected. Notice the presence of some vacuolations (V). (H \& E x 400) 


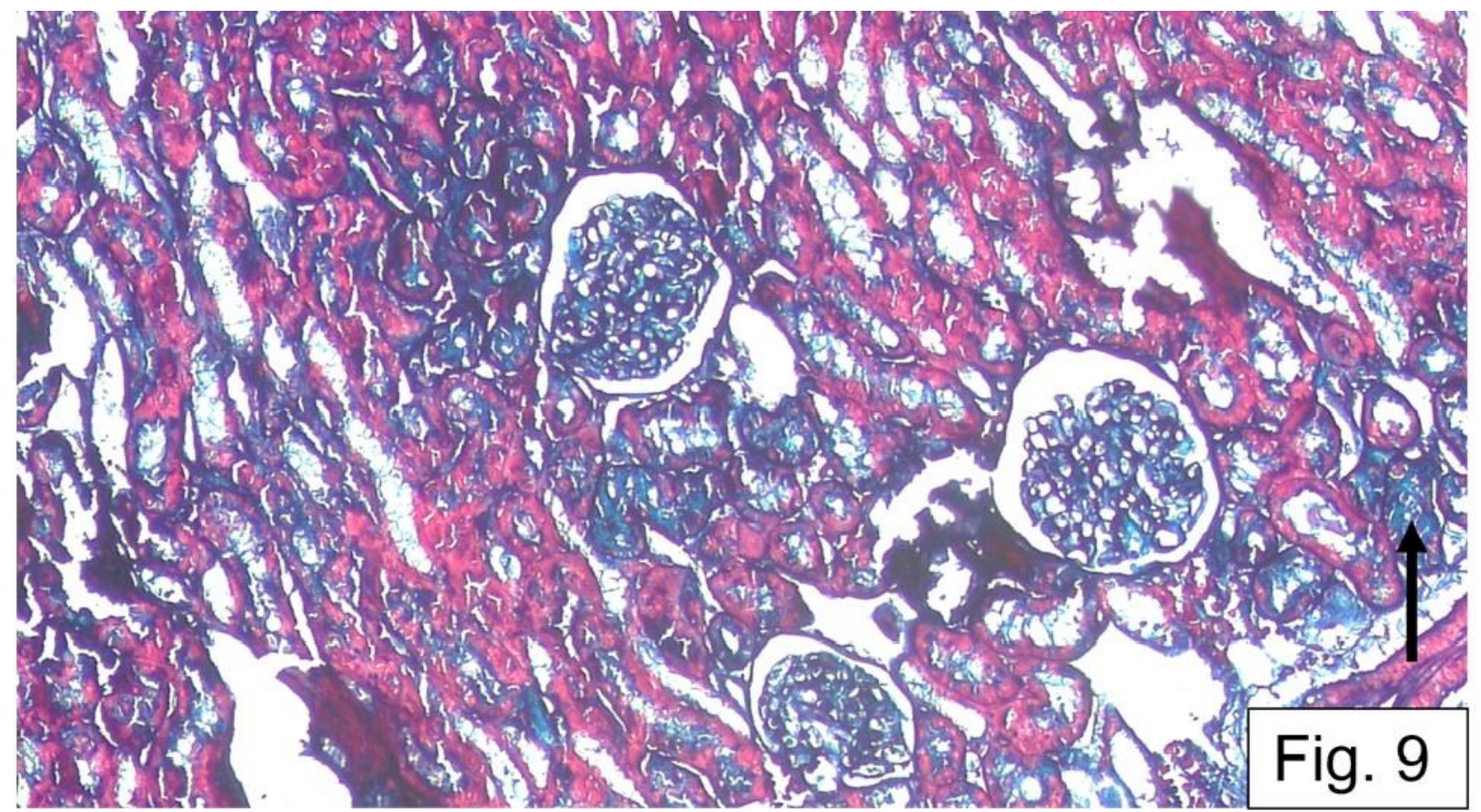

Fig. (9): A photomicrograph of a section of the renal cortex of group (III) (cisplatin+ spirulina-treated group) showing minimal amount of the collagen fiber in the renal interstitial (black arrow).

(Masson's Trichrome x 400)

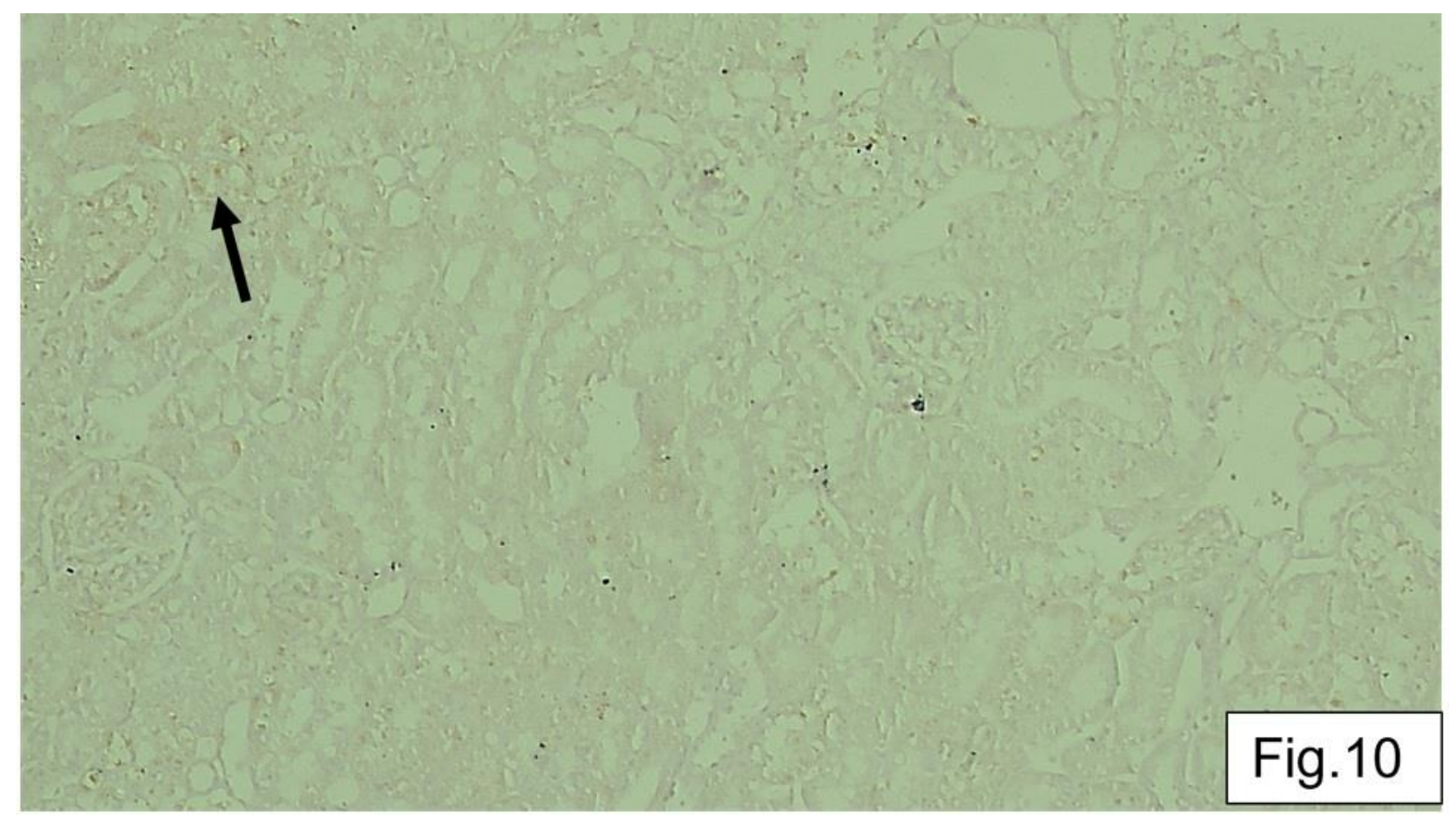

Fig. (10): A photomicrograph of a section of the renal cortex of group (III) (cisplatin+ spirulina-treated group) showing that spirulina significantly decreased the expression of active caspase-3, which induced by cisplatin administration. There were a few focal areas of tubular necrosis with presence of few apoptotic cells appeared as brownish cytoplasmic staining. (Caspase-3 X400) 
https://ejhm.journals.ekb.eg/

\section{DISCUSSION}

There is no adequate therapy for nephrotoxicity induced by $\mathrm{CP}$ in humans and many antioxidants have been investigated in order to alleviate the impact of this nephrotoxicity. Even though the majority of them demonstrated efficacy in experimental models, unfortunately none of them demonstrated definite efficacy in humans ${ }^{(23,24)}$.

Our results showed that tubular necrosis as well as vascular component atrophy in glomerulus in rats treated with cisplatin. These findings are compatible with those obtained by Ravindra et al. ${ }^{(25)}$.

The decrease in the side effects of chemotherapeutic agents was important for the success of cancer treatment. Spirulina platensis (Sp) were investigated to alleviate the toxic impact of chemotherapeutic medications via its Reno protective effectiveness.

Cisplatin treated group demonstrated an extensive amount of collagen fibers, which are distributed mainly around the renal tubules and within the glomeruli. This result is in accordance with El-Sayed $\boldsymbol{e t}$ al. ${ }^{(26)}$ who revealed a substantial elevation in caspase- 3 activity in rats treated with cisplatin.

Apoptosis contributes to the pathogenesis of a wide range of renal diseases. Cisplatin-induced nephrotoxicity is mediated by caspase-3 dependent as well as independent apoptotic pathways (27). After cisplatin treatment many studies reported an elevation in serum creatinine as well as the nitrogen of blood urea ${ }^{(28)}$. Spirulina pretreatment diminished serum creatinine and BUN triggered by cisplatin, and this is more effective compared to administrating spirulina after injecting cisplatin ${ }^{(29)}$.

The mechanisms of spirulina in the different biological actions are varied. Its mechanism as anticancer is contributed to its content of endonuclease that repairs the damage in DNA. As antiviral due to its calcium sulfated polysaccharide that inhibits viruses' in-vitro replication. Its action as an antimicrobial agent is due to its fatty acids (unusually elevated content of $\gamma$-linolenic acid), and its metal protective role, which may indicate the presence of vitamins $\mathrm{C}$ and $\mathrm{E}$, beta-carotene, selenium as well as phycocyanin ${ }^{(30)}$. Spirulina is an antioxidant agent, which may have inhibited the cisplatin free radicals, before affecting kidney function. The findings of Kuhad et al. ${ }^{(31)}$ align with our findings. They examined the decrease of the oxidative stress in cisplatin-treated rats after spirulina treatment. The findings of the present study detected that cisplatin induced substantial renal oxidative as well as markedly degeneration in the renal function and these modifications in the function of the kidney, which was detected in the rat system were correlated with the nephrotoxic impacts of cisplatin in men ${ }^{(32)}$. Many recorded studies reported that spirulina could be used within the drugs to reduce their side effects on the metabolism and to increase the efficiency of the immune system of the body. It has been detected that spirulina is featured by anti-inflammatory properties, via inhibiting the release of histamine from mast cell-mediated allergic reactions ${ }^{(33)}$.

Cp-treated kidneys demonstrated substantial histological alternations, particularly in the outer cortex as compared to the kidneys from controls. Spirulina triggered these histological renal alternations, with potential ROS involvement. Spirulina pretreatment protected the kidneys before cisplatin injection ${ }^{(34)}$. The phycocyanin pigment in spirulina may be the cause of its protective impact. The antioxidant effect of spirulina could be the cause of renoprotection. The clinical manifestations of $\mathrm{CP}$ range from an asymptomatic increase in serum creatinine to renal dysfunction and it was illustrated the protective impacts of spirulina on kidney against nephrotoxicity induced by cisplatin ${ }^{(34)}$.

\section{CONCLUSION}

Cisplatin resulted in histological as well as biochemical changes in the kidney of adult male rats. Administration of spirulina with cisplatin attenuated these negative impacts which can be attributed to the antioxidant activity.

\section{REFERENCES}

1. Moncharmont $\mathrm{C}$, Auberdiac $\mathrm{P}$, Melis A et al. (2011): Cisplatin or carboplatin, that is the question. Bull. Cancer, 98: 164-175.

2. Yao X, Panichpisal K, Kurtzman $\mathbf{N}$ et al. (2007): Cisplatin nephrotoxicity:a review. Am. J. Med.Sci., 334: 115-124.

3. Uccelli A, Moretta L and Pistoia V (2008): Mesenchymal stem cells in health and disease. Nature Reviews Immunology, 8 (9): 726-736.

4. Chawla L, Eggers P, Star R et al. (2014): Acute kidney injury and chronic kidney disease as interconnected syndromes. The New England Journal of Medicine, 371 (1): 58-66.

5. Karasawa $\mathbf{T}$ and Steyger $\mathbf{P}$ (2015): An integrated view of cisplatin-induced nephrotoxicity and ototoxicity. Toxicology letters, 237: 219-227

6. Akcay A, Turkmen K, Lee D et al. (2010): Update on the diagnosis and management of acute kidney injury. International journal of nephrology and renovascular disease, 3: 129-140.

7. Fuertes M, Alonso C and Pérez J (2003): Biochemical modulation of Cisplatin mechanisms of action: enhancement of antitumor activity and circumvention of drug resistance. Chemical reviews, 103: 645-662.

8. Mukhopadhyay P et al. (2010): Cannabinoid-2 receptor limits inammation, oxidative/nitrosative stress, and cell death in nephropathy. Freeradical biology \& medicine, 48: 457-467. 
9. Khan Z, Bhadouria P, Bisen P (2005): Nutritional and therapeutic potential of Spirulina. Curr Pharm Biotechnol., 6:373-379.

10. Remirez D, Gonzalez R, Merino N et al. (2002): Inhibitory effects of Spirulina in zymosan-induced arthritis in mice. Mediators Inflamm., 11: 75-79.

11. Khan M, Shobha J, Mohan I et al. (2005): Protective effect of Spirulina against doxorubicin-induced cardiotoxicity. Phytother Res., 19: 1030-1037.

12. Chu W, Lim Y, Radhakrishnan A et al. (2010): Protective effect of aqueous extract from Spirulina platensis against cell death induced by free radicals. BMC Complement Altern Med., 10 (1): 53.

13. Rodríguez-Salgueiro $S$, Ramírez-Carmenate $Z$, González-Núñez L (2017): An update on potential applications of Spirulina sp. and C-phycocyanin to treat kidney diseases. Ann Res Antioxid., 2:1.

14. Wu Q, Liu L, Miron A et al. (2016): The antioxidant, immunomodulatory, and anti-inflammatory activities of Spirulina: an overview. Arch Toxicol., 90 (8): 1817-1840.

15. Hemalatha $\mathrm{K}$, Pugazhendy $\mathrm{K}$, Jayachandran $\mathrm{K}$ et al. (2021): Studies on the protective efficacy of Spirulina against lead acetate induced hepatotoxicity in Rattus norvegicus. Int J Chem Anal Sci., 3:1509-1512.

16. Gutiérrez-Salmeán G, Fabila-Castillo L, ChamorroCevallos G (2015): Aspectos nutricionales y toxicológicos de Spirulina (arthrospira). Nutricion Hospitalaria, 32 (1): 34-40.

17. Zimmermann M (1983): Ethical guidelines for investigations of experimental pain in conscious animals. Pain,16: 109-110.

18. EL-Tantawi H, Abozeid F (2019): Impact of Spirulina on Propylthiouracil- Induced Hypothyroidism in Albino Rats, A Histological, Immunohistochemical and Biochemical Approach. Egyptian Journal of Histology, 42(4): 849-860.

19. El-Sayed E, Abdallah A, Mansour A et al. (2015): Thymol and carvacrol prevent Cisplatin-induced nephrotoxicity by abrogation of oxidative stress, inflammation, and apoptosis in rats, $\mathrm{J}$ Biochem Mol Toxicol., 29:165.

20. Iranpour F, Kheiri S (2016): Coadministration of calcium chloride with lead acetate can improve motility of cauda epididymal spermatozoa in Swiss white mice. Int J Reprod BioMed., 14 (2): 141-144.

21. Suvarna S, Layton C and Bancroft J (2013): The Hematoxylin and eosin. Theory Practice of histological techniques. 7th ed. Ch. 10 and 11. Philadelphia: Churchill Livingstone of El Sevier, Pp:179-220.

22.Stenberg L, Kanje M, Dolezal K et al. (2012): Expression of activating transcription factor 3 (ATF 3 ) and caspase 3 in Schwann cells and axonal outgrowth after sciatic nerve repair in diabetic BB rats. Neuroscience letters, 515 (1): 34-38.

23. Perazella M, Moeckel G (2010): Nephrotoxicity from chemotherapeutic agents: clinical manifestations, pathobiology, and prevention/therapy. Semin. Nephrol., 30: 570-581.

24. Nemmar A, Al-Salam S, Zia S et al. (2010): Diesel exhaust particles in the lung aggravate experimental acute renal failure. Toxicol. Sci., 113: 267-277.

25. Ravindra P, Bhiwgade D, Kulkarni S et al. (2010): Cisplatin induced histological changes in renal tissue of rat. J Cell Anim Biol., 4 (7): 108-11.

26. El-Sayed M, Mansour A, Ghobara M (2015): Abrogation of cisplatin-induced nephrotoxicity in rats by lycopene through ameliorating oxidative stress, inflammation, and apoptosis. International Journal of Therapeutic Applications, 27: 16-22.

27. Cummings B, Schnellmann R (2002): Cisplatin induced renal cell apoptosis: caspase 3- dependent and independent pathways, J Pharmacol Exp Ther., 302: 8.

28. Palipoch S, Punsawad C, Koomhin P et al. (2014): Hepatoprotective effect of curcumin and alpha-tocopherol against cisplatin-induced oxidative stress. BMC Complement Altern Med., 14 (1): 111.

29. Shimeda Y, Hirotani Y, Akimoto Y et al. (2005): Protective effects of capsaicin against cisplatin-induced nephrotoxicity in rats. Biol Pharm Bull., 28 (9): 16351638.

30. Hoseini, S, Khosravi-Darani K, Mozafari M (2013): "Nutritional and medical applications of spirulina microalgae." Mini reviews in medicinal chemistry,13 (8): 1231-1237.

31. Kuhad A, Tirkey N, Pilkhwal S et al. (2006): Reno protective effect of Spirulina fusiformis on cisplatininduced oxidative stress and renal dysfunction in rats. Ren Fail., 28 (3): 247-254.

32. Segelov E, Mann G, Harnett P (1998): Mechanisms determining sensitivity to cisplatin in three mutant Chinese hamster ovary cell lines. Mutat Res/DNA Repair, 407 (3): 243-252.

33. Mao T, Van de Water J, Gershwin M (2005): "Effects of a Spirulina-based dietary supplement on cytokine production from allergic rhinitis patients." Journal of Medicinal Food, 8 (1): 27-30.

34. Zakaria M, El-Tantawy F, Khater S et al. (2019): Protective and curative role of Spirulina platensis extracts on cisplatin induce acute kidney injury in rats. Egyptian Journal of Basic and Applied Sciences, 6 (1): 54-67. 